\title{
Prevalence of Helminths in Digestive Tract of Cows in Indonesia
}

\author{
Carolina Erfinda Puspita Purwandani ${ }^{1}$, Suryo Kuncorojakti ${ }^{1 *}$, and Lucia Tri Suwanti ${ }^{2}$ \\ ${ }^{I}$ Department of Veterinary Anatomy, Faculty of Veterinary Medicine, Universitas Airlangga, Surabaya, East Java, Indonesia \\ ${ }^{2}$ Department of Veterinary Parasitology, Faculty of Veterinary Medicine, Universitas Airlangga, Surabaya, East Java, Indonesia \\ *Corresponding Author's Email: suryokuncorojakti@fkh.unair.ac.id; (DORCiD: 0000-0001-9332-2663
}

\begin{abstract}
Helminthiasis is an important problem that affects the productivity and health of cows in tropical countries. This study aimed to examine the prevalence of helminthiasis in the digestive tract and the factors that affect it. In the current study, feces samples were from 75 cows aged up to one year old. The samples were collected from areas with the majority of the population as cattle breeders. The data were analyzed descriptively and statistically. The result indicated that most of the cows have suffered from helminthiasis caused by Toxocara vitulorum and the prevalence of intestinal helminths was mostly mild. Toxocara vitulorum spp. was the most common nematode worm that infected cows. The most effective factors on prevalence of helminthiasis in cows were age and cage location.
\end{abstract}

Keywords: Cow, Helminthiasis, Gastrointestinal, Prevalence

\section{INTRODUCTION}

Helminthiasis is a big problem for livestock, especially for cattle farming, in Indonesia. This type of ruminant is at risk of being exposed to parasitic worms through the digestive tract. This infection can occur in many areas, especially in tropical climates. Most of the infectious worms belong to the Trematodes, Cestodes, and Nematodes (Raza et al., 2012). These parasites affect livestock productivity and human health. Indirect losses related to infection include a reduction in the potential of productivity. Some cases present the final stage of the infection, such as a decrease in growth rate, weight loss, decrease in the quality of meat, skin, offal, reduction of livestock productivity, such as milk production and the occurrence of diarrhea, anorexia, and sometimes anemia (Stromberg et al., 2015). In chronic conditions, this disease could lead to emaciation, weakness, and reduced production power but does not cause death. However, it is Mild to moderate worm infections do not always reveal tangible clinical symptoms, whereas severe infections from adult worms could cause digestive disorders and stunted growth in young livestock (Subekti et al., 2010).

The various disease-causing factors vary from one species to another. The spread of worm infections is quite high in the humid and hot tropics, the situation which supported the survival of these worms. The susceptibility of the animal to worm infection was affected by intrinsic factors of the animal's body which included animal's species, sex, age, condition, and animal's immune system ability (Charlier et al., 2016).

In 2015, there were 3429 cows in the study area included 1848 male and 1581 female ones. Most breeders in the area had 1-5 livestock per household (BPS Kabupaten Magelang, 2016). However, farmers still do not pay attention to sanitation and hygiene in raising livestock, especially those who raise cattle behind the house with semi-permanent cage building conditions with no feces and urine drainage. Poor livestock management and poor cage sanitation would allow parasitic infectious agents to enter easily through the contamination of feed and drinking.

The purpose of the current study was to study the prevalence of gastrointestinal helminthiasis in cows in areas with a majority of the population as cattle breeders.

\section{MATERIALS AND METHODS}

All experimental protocols and procedures were approved by the Institutional Animal Care of Indonesia. This experimental study took 8 weeks in Magelang District, Central Java, Indonesia. Feces samples were randomly selected from a total number of 75 cows aged up to one-year-old. The samples were then examined at the Parasitology Laboratory, Faculty of Veterinary, Universitas Airlangga, Surabaya, Indonesia. The presence of worm eggs showed a positive sample.

Sample examination was carried out using the Natif method, simple sedimentation method, and fulleborn float method. The examinations were carried out on a microscope with 100× magnification in all methods except for the 
fulleborn method which used $100 \times$ and $400 \times$ magnification. The Natif method was done by liquefying 1 gram of stool with one or two drops of water until it became homogeneous. The simple sedimentation method was done by adding water to the stool with a ratio of 1 gram:10 $\mathrm{ml}$ to the plastic (Sample container) until it was evenly distributed and filtered. The filter was centrifuged for 5 minutes at a speed of $1500 \mathrm{rpm}$ until the supernatant was clear and then one drop was taken. The fulleborn float method was carried out by making a stool suspension, centrifuging it for $3 \mathrm{x}$ at a speed of $1500 \mathrm{rpm}$ for 5 minutes then 3 minutes, and finally 5 minutes with a pause between centrifugation, i.e. removing the supernatant and making sure the supernatant was clear. In the third stage, the remaining supernatant was added to a saturated sugar solution of up to $1 \mathrm{~cm}$ first, then centrifuged for stage three. The liquid would form a convex surface at the mouth of the centrifuge tube. Furthermore, it was covered with a lid and left for 2 minutes.

If a positive sample was found then the calculation of fecal Eggs Per Gram (EPG) was calculated through the Lucient Brumpt method. The examination was carried out using a 100 times magnification microscope. The data were obtained, and the prevalence and statistical analysis of tree regression using the SPSS (version 23) program 23.0 for Windows were performed.

\section{RESULTS AND DISCUSSION}

This study found worm eggs in $57(76 \%)$ cow feces samples. The cows were declared infected with worms and stated as positive samples. About 18 (24\%) were stated negatively from worm eggs. The results found that different types of worms, including Strongyloides spp., Moniezia dances, Toxocara vitulorum, Trichuris spp., And Oesophagostomum spp. play a role in infections. The types of worms that caused infection are indicated in Table 1.

Table 1. Percentage of worm types in infected cattle aged one-year-old

\begin{tabular}{lcc}
\hline Types of worms & N & Percentage (\%) \\
\hline Toxocara vitulorum & $20 / 75$ & 26.7 \\
Strongyloides spp. & $16 / 75$ & 21.3 \\
Moniezia expansa & $14 / 75$ & 18.7 \\
Trichuris spp. & $1 / 75$ & 1.3 \\
Oesophagostomum spp. & $1 / 75$ & 1.3 \\
Strongyloides spp. and Toxocara vitulorum & $3 / 75$ & 4 \\
Toxocara vitulorum and Moniezia expansa & $1 / 75$ & 1.3 \\
Strongyloides spp., Toxocara vitulorum and Moniezia expansa & $1 / 75$ & 1.3 \\
\hline Total & 57 & 76 \\
\hline
\end{tabular}

$\mathrm{N}$ : Number

The number of samples and types of infections described the pattern of worm infections. The highest number of Toxocara vitulorum worm eggs was 24 positive samples that included 20 single infection samples and four mixed infections. Toxocara vitulorum worm eggs were found through examination with Natif, sedimentation, and floating methods. Toxocara vitulorum eggs size were $83.42 \mu \mathrm{m} \times 68.54 \mu \mathrm{m}$. Positive samples of Strongyloides spp. were 20 . Worm eggs were found in 16 single infection samples and 4 mixed infections. The egg was oval-shaped, with an egg size of $45.70 \mu \mathrm{m} \times 25.94 \mu \mathrm{m}$. Moniezia expanza worm eggs were found in 16 positive samples, 14 single infections and 2 mixed infections. Moniezia expanza worm eggs were found both through sedimentation and floating examinations. Moniezia expanza eggs were triangular and had a size of $56.78 \mu \mathrm{m}$.

In addition to the three types of worms, two worms had similar prevalence ( 1 positive sample), which were worm eggs of Trichuris spp. and worm eggs of Oesophagostomum spp. Worm eggs of Trichuris spp. were found only by the floating method. Eggs were oval like lemons and had transparent plugs at both ends. The egg size was $78.9 \mu \mathrm{m} \times 40.1$ $\mu \mathrm{m}$. Oesophagostomum spp. was only found by the floating method. Eggs were elliptical with thin membranes and a size of $87 \mu \mathrm{m} \times 44 \mu \mathrm{m}$.

This type of infection did not occur with a single infection. Stool examination results also indicated mixed infections. Infection with mixed worm eggs of Strongyloides spp. and Toxocara vitulorum was found in three positive samples and this mixed infection was found in sedimentation and floating methods. Infection of a mixture of Toxocara vitulorum and Moniezia expansa worm eggs was found in one positive sample and was only observed in the floating method. Infection of a mixture of Toxocara vitulorum, Strongyloides spp., and Moniezia expansa was observed in a positive sample using the floating method. Figure 2 demonstrates a single infection and a mixture of worm eggs based on a magnification of a $100 \times$ microscope and there was a black arrow. The figures show these worm eggs in order as Toxocara vitulorum worm eggs (A), Strongyloides spp. worm eggs (B), Moniezia expansa worm eggs (C), Trichuris spp. worm eggs (D), and Oesophagostomum spp. worm eggs (E). 

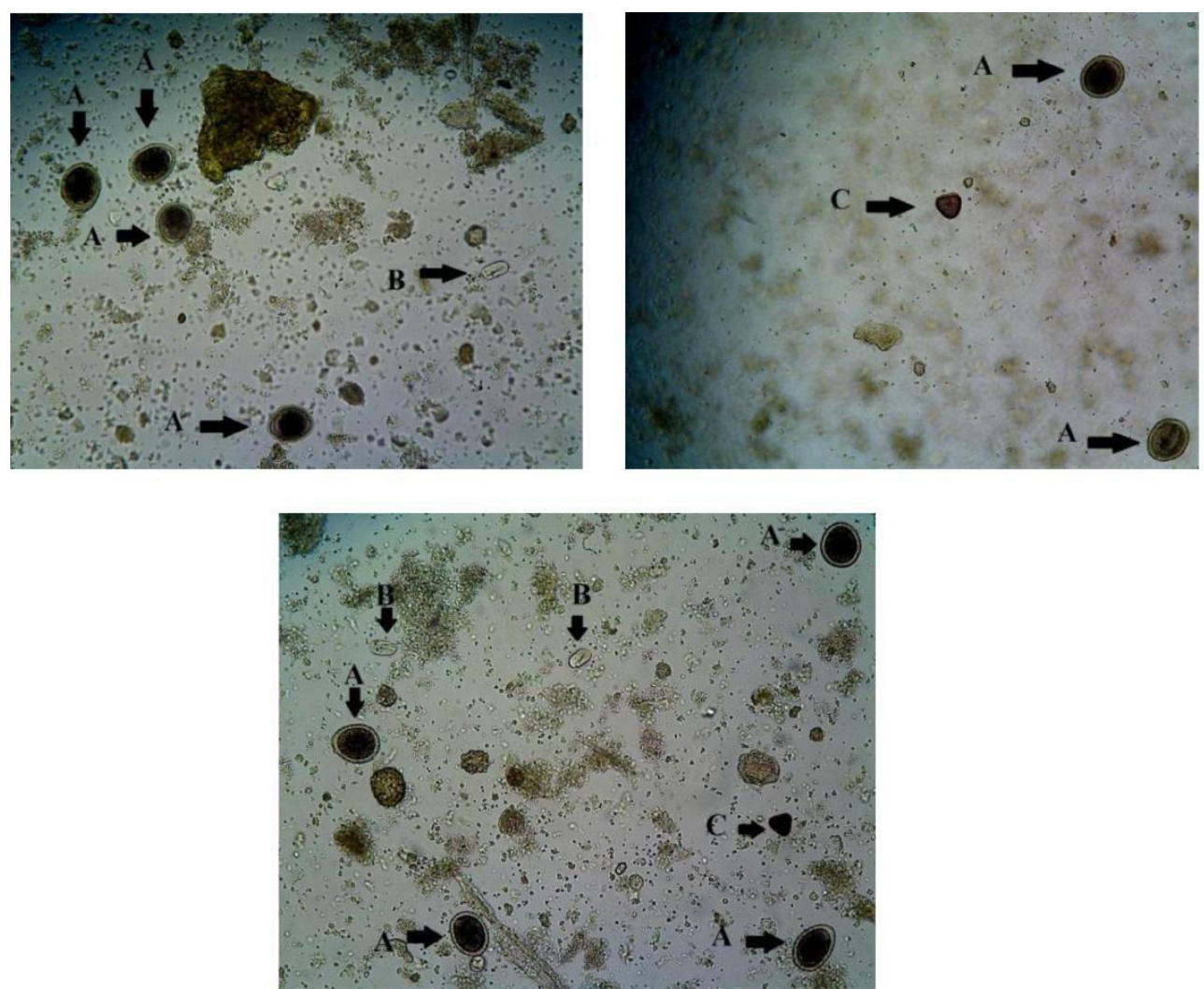

Figure 1. Worm eggs in infected cattle aged one-year-old.
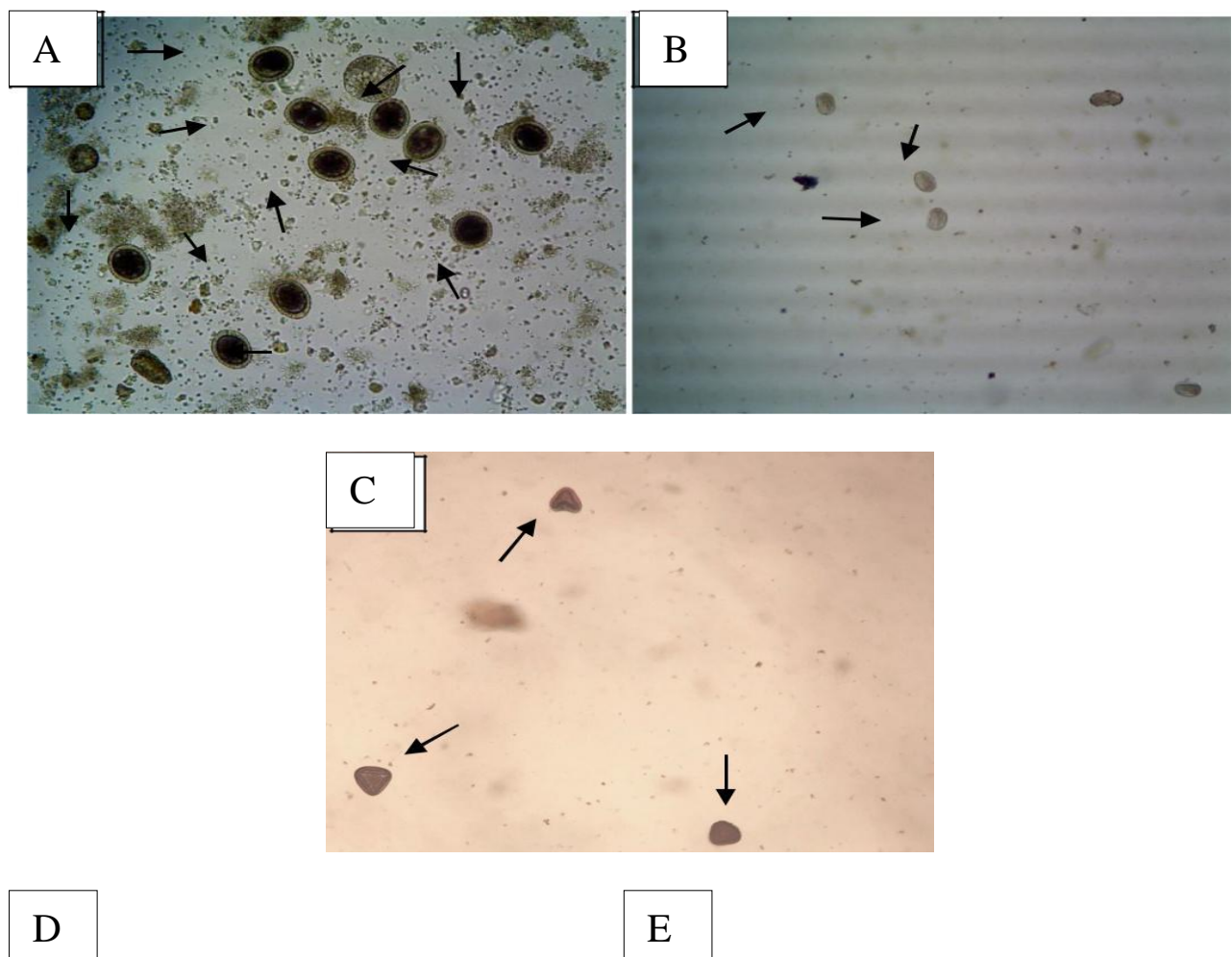

Figure 2. Infection of infected cattle with a mixture of Toxocara vitulorum (arrow A), Strongyloides spp. (arrow B), and Moniezia expansa (arrow C) eggs

Tree regression analysis indicated that the prevalence of digestive tract helminthiasis in cattle occurred due to cage location. Cows less than 7.5 months old had a higher prevalence of worm infections by $70.2 \%$ (40 of 57 positive samples) than cows over 7.5 months old which had a prevalence of worm infections by $94.4 \%$ (17 of 18 samples positive). The prevalence of worm infections was $77.5 \%$ (31 out of 40 positive samples). On the one hand, cows aged more than 5 months had the prevalence of worm infections as much as $52.9 \%$ ( 9 of 17 positive samples). Cows older than 5.5 months consisted of cows less than 6.5 months had the prevalence of worm infections by $46.2 \%$ (6 of 13 
positive samples). However, cows aged more than 6.5 months had a prevalence of worm infection by $75 \%$ ( 3 from 4 positive samples). Cows less than 6.5 months old were affected by the location of the cage. Cows in the lowlands had the prevalence of worm infections as much as $62.5 \%$ (five positive samples), which was higher than cows in the highlands with the prevalence of worm infections as much as $20 \%$ (One positive sample).

Calculation of mean EPG with Lucient brumpt method in cattle fecal samples indicated that the number of worm eggs from each sample has been calculated per gram of stools. There were two categories of infections, first was a mild infection which was found in 55 samples of 57 positive samples and occurred in the highlands and lowlands with an average of 230.5. The second was a moderate infection that was found in 2 samples and only occurred in the lowlands with an average of 740 .

\section{DISCUSSION}

Most feces samples of cows aged 0-1-year-old were positive for worm eggs. Genus DNA extraction process plays a major role in the identification of Polymerase Chain Reaction (PCR) because it affects the transmission process (Stromberg et al., 2015). Worms can live and develop through certain food elements. Herbivorous and carnivorous hosts consumed animal feed that might be contaminated by infective larvae. This infection will stop if a host can eradicate the pre-infection stage. The direct transfer of host-to-host in worm infections is limited to several parasites, for example through prenatal infection from the mother to a growing embryo and for transmission through skin penetration. Some filarial worms were transmitted by vectors (Bain and Babayan, 2002).

Age and cage factors influence the prevalence of helminthiasis. The size of the parasitic eggs between adult cows and calves was different. The number of nematode eggs per gram found in cow feces in grasslands had heritability so that prevention and control of parasites could be carried out (Jenny et al., 2016). Species concentration, prevalence, and abundance of parasites have a relationship with the age of the host, gender, food, and season (Ibrahim et al., 2008).

Weaning calves are done when a calf is over 7.5 months old. Many cattle aged less than 7.5 months old were infected with worms, and the results of the examination indicated that the most worm egg infections are Toxocara vitulorum. Calves can be infected through colostrum. When a calf is 5 months old, worms might be released spontaneously (Lovera et al., 2017).

Cows that are less than 5.5 months old had more positive samples, which were 31 samples aged more than 5.5 months with only 9 positive samples. Cows younger than 6 months were not free of helminthiasis because of several factors, including immunity which is still passive until the age of three weeks so cattle can be infected with pathogenic agents (parasites, bacteria, viruses, fungi, and protozoa). Food with poor quality is also a factor that plays a role in being infected by different infections. Food intake and stress levels affect calf immune status and weaning readiness. The optimal health and well-being of calves at an early stage could cause improvement in their long-term health and wellbeing (Hulbert and Moisá, 2016). Cows older than 5.5 months are consisted of cows more than 5.5 months and younger than 6.5 months (between 5.5 and 6.5 months). Cows younger than 6.5 months old were affected by the location of the cage. Cows in the lowlands indicated positive results, compared to cows in the highlands, due to the humidity and temperature factors that are optimal for the development of worm egg larvae.

The type of identified eggs was Toxocara vitulorum, which were $83.42 \mu \mathrm{m} \times 68.54 \mu \mathrm{m}$ in size and round in shape. The Toxocara vitulorum eggs are subglobular in shape surrounded by albumin layers that are thick and $75-95 \times$ 60-75 $\mu \mathrm{m}$ in size (Subekti et al., 2010). The molecular characteristics of worm eggs should be identified to determine the zoonosis and dynamics of transmission (Squire et al., 2017). Humans, especially children, often swallow these eggs accidentally and become infected. Infections in humans, in contrast to their definitive hosts, remain hidden, often resulting in diseases caused by migrating larval stages. Visceral Larvae Migrants and Ocular Migrant Larvae are two clinical manifestations that produce definite syndromes and appear as serious health problems so that the diagnosis and treatment of both would be difficult (Despommier, 2003). A pair or several types of parasitic worms can infect one host, resulting in multi-host aggregation. Nearly $50 \%$ of individuals have at least one species of worm, but some certain species show a higher prevalence than others. Parasitic sharing patterns are likely to arise from shared space use through mixed-species aggregation (VanderWaal et al., 2014).

Helminthiasis still has no treatment and from the results of interviews with these farmers, there has never been a worm treatment even though the high circulation of zoonotic pathogens is related to public health in livestock breeding areas. All pathogenic infections increase with a large number of hosts (Lovera et al., 2017). A low to moderate number of stool eggs from livestock indicated that worm infections in this region were still under control. Cow farmers were encouraged to continue good animal husbandry practices that ensure low worm infection rates. However, small farmers were encouraged to control worm infections in their sheep and goats with anthelmintic treatment. In addition, breeders also need to consider the use of inputs (feed, pastures, medicines) and aspects that affect the infection process (Charlier et al., 2014). The joint role for animal management and anthelmintic therapy has considered the use of anthelmintic 
therapy to effectively control parasitic nematodes since some anthelmintic programs did not work according to the program (Stromberg et al., 2015).

\section{CONCLUSION}

Toxocara vitulorum spp. is the most common nematode worm that infects cows of the Magelang region of Indonesia. The factors that influence the prevalence of cow helminthiasis are age and cage location. Small farmers were encouraged to control worm infections in their sheep and goats with anthelmintic treatment. In addition, breeders also need to consider the use of inputs (feed, pastures, medicines) and aspects that affect the infection process in the studied region.

\section{DECLARATIONS}

\section{Competing interests}

Authors declare no competing interests.

\section{Authors' contribution}

The authors have participated in this study equally.

\section{Ethical consideration}

Ethical issues (Including plagiarism, consent to publish, misconduct, data fabrication and/or falsification, double publication and/or submission, and redundancy) have been checked by the authors.

\section{REFERENCES}

Badan Pusat Statistik (BPS) Kabupaten Magelang (2016) Publikasi Statistik Daerah, Indonesia.

Bain O, and Babayan S (2003). Behaviour of filariae: morphological and anatomical signatures of their life style within the arthropod and vertebrate hosts. Filaria journal, 2(1): 16. DOI: https://doi.org/10.1186/1475-2883-2-16

Charlier J, Voort MVD, Kenyon F, Skuce P, and Vercruysse J (2014) Chasing helminths and their economic impact on farmed ruminants. Trends in Parasitology, 30(7): 361-367. DOI: https://doi.org/10.1016/j.pt.2014.04.009 .

Charlier J, Waele VD, Ducheyne E, Voort MVD, Velde FV, and Claerebout E (2016) Decision making on helminths in cattle: Diagnostics, economics and human behaviour. Irish Veterinary Journal, 69(1): 1-14. DOI: https://doi.org/10.1186/s13620-0160073-6

Despommier D (2003) Toxocariasis: Clinical Aspects, Epidemiology, Medical Ecology, and Molecular Aspects. American Society for Microbiology, 16(2): 265-272. DOI: https://doi.org/10.1128/CMR.16.2.265-272.2003.

Hulbert LE, and Moisa SJ (2016) Stress, immunity, and the management of calves. Journal of Dairy Science, 99(4): 3199-3216. DOI: https://doi.org/10.3168/jds.2015-10198.

Ibrahim MM, and Ghamdi MGM (2008) Helminths community of veterinary importance of livestock in relation to some ecological and biological factors. Turkiye Parazitoloji Dergisi, 32(1): 42-47. Available at: http://parazitoloji.dergisi.org/pdf/pdf_TPD_319.pdf

Chaparro JJ, Ramírez NF, Villar D, Fernandez JA, Londoño J, Arbeláez C, López L, Aristizabal M, Badel J, Palacio LG, et al. (2016). Survey of gastrointestinal parasites, liver flukes and lungworm in feces from dairy cattle in the high tropics of Antioquia, Colombia. Parasite Epidemiology and Control, 1(2): 124-130. DOI: https://doi.org/10.1016/j.parepi.2016.05.001

Lovera R, Fernandez MS, Jacob J, Lucero N, Morici G, Brihuega B, Farace MI, Caracostantogolo J, and Cavia R (2017). Intrinsic and extrinsic factors related to pathogen infection in wild small mammals in intensive milk cattle and swine production systems. PLoS Neglected Tropical Diseases, 11(6): 1-20. DOI: https://doi.org/10.1371/journal.pntd.0005722.

Raza MA, Bachaya HA, Akhtar MS, Arshad HM, Murtaza S, Ayaz MM, Naeeem M, and Basit A (2012). Point Prevalence of Gastrointestinal Helminthiasis in Buffaloes (Bubalus bubalis) at The Vicinity of Jatoi, Punjab, Pakistan. Science International (Lahore), 24(4): 465-469. Available at: http://www.sci-int.com/pdf/88120633525-buffalo\%20research\%20paperRaza\%2024$4[1] \% 20 \% 20 . p d f$

Squire SA, Yang R, Robertson I, Ayi I, and Ryan U (2017). Molecular characterization of Cryptosporidium and Giardia in farmers and their ruminant livestock from the Coastal Savannah zone of Ghana. Infection, Genetics and Evolution, 55: 236-243. DOI: 1 https://doi.org/10.1016/j.meegid.2017.09.025

Stromberg BE, Gasbarre LC, Ballweber LR, Dargatz DA, Rodriguez JM, Kopral CA, and Zarlenga DS (2015). Prevalence of internal parasites in beef cows in the United States: Results of the national animal health monitoring system's (NAHMS) beef study, 2007-2008. Canadian Journal of Veterinary Research, 79(4): 290-295. PMID: 26424909

Subekti, S, Mumpuni S, Koesdarto S, and dan K HP (2010) Buku Ajar Helmintologi Veteriner. Surabaya: Airlangga University Press.

VanderWaal K, Omondi GP, and Obanda V (2014). Mixed-host aggregations and helminth parasite sharing in an East African wildlife-livestock system. Veterinary Parasitology, 205(1-2): 224-232. DOI: https://doi.org/10.1016/j.vetpar.2014.07.015 